\title{
The Relationship Between Maximum Isometric Strength and Ball Velocity in the Tennis Serve
}

\author{
by \\ Ernest Baiget ${ }^{1}$, Francisco Corbi², Juan Pedro Fuentes ${ }^{3}$, Jaime Fernández-Fernández ${ }^{4}$
}

The aims of this study were to analyze the relationship between maximum isometric strength levels in different upper and lower limb joints and serve velocity in competitive tennis players as well as to develop a prediction model based on this information. Twelve male competitive tennis players (mean $\pm S D$; age: $17.2 \pm 1.0$ years; body height: $180.1 \pm 6.2 \mathrm{~cm}$; body mass: $71.9 \pm 5.6 \mathrm{~kg}$ ) were tested using maximum isometric strength levels (i.e., wrist, elbow and shoulder flexion and extension; leg and back extension; shoulder external and internal rotation). Serve velocity was measured using a radar gun. Results showed a strong positive relationship between serve velocity and shoulder internal rotation $(r=0.67 ; p<0.05)$. Low to moderate correlations were also found between serve velocity and wrist, elbow and shoulder flexion - extension, leg and back extension and shoulder external rotation $(r=0.36-0.53 ; p=0.377-0.054)$. Bivariate and multivariate models for predicting serve velocity were developed, with shoulder flexion and internal rotation explaining $55 \%$ of the variance in serve velocity $(r=0.74 ; p<0.001)$. The maximum isometric strength level in shoulder internal rotation was strongly related to serve velocity, and a large part of the variability in serve velocity was explained by the maximum isometric strength levels in shoulder internal rotation and shoulder flexion.

Key words: serve velocity, isometric testing, shoulder internal rotation.

\section{Introduction}

Nowadays, the serve stroke is the most important shot in competitive tennis, allowing the player to win the point directly through an ace or dominate the rally since the beginning (Gillet et al., 2009; Kovacs and Ellenbecker, 2011b). This stroke involves a patterned, repetitive motion that is kinetically linked, and needs to be performed with appropriate technique (Jayanthi and Esser, 2013). From a biomechanical perspective, the tennis serve needs to activate all components of the kinetic chain (i.e., feet, lower limbs, trunk, shoulder, elbow, wrist and hand) (Bonato et al., 2014; Eygendaal et al., 2007). Competitive players should train physical aspects like strength, speed, power, flexibility, local muscular endurance and muscular balance, which could potentially reduce the risk of injury (Kovacs and Ellenbecker, 2011b; Reid and Schneiker, 2008). Specifically, strength training has become vital in contemporary tennis as the velocity and power deployed in the game continue to increase (Abrams et al., 2011; Cardoso Marques, 2005). Strength training programs involving different methods (e.g., elastic tubing, medicine ball exercises, resistance training or lightweight dumbbell training) have been shown to increase serve velocity in elite male junior tennis players and in male and female college tennis players (Fernandez-Fernandez et al., 2013; Kraemer et al., 2003; Treiber et al., 1998).

The relationship between strength levels

\footnotetext{
1 - Sport Performance Analysis Research Group, University of Vic - Central University of Catalonia, Barcelona, Spain.

2 - Insitut Nacional d'Educació Física de Catalunya, University of Lleida, Lleida, Spain.

3 - Sports Sciences Faculty, University of Extremadura, Cáceres, Spain.

4 - Sports Research Centre, Miguel Hernandez University, Elche, Spain.
} 
and serve velocity has been reported using isokinetic testing (Cohen et al., 1994; Pugh et al., 2003; Signorile et al., 2005), but there is little information on the relationships between maximum isometric strength and ball velocity. Due to mechanical and neural significant differences between dynamic and static muscular actions, the use of isometric tests has been considered inappropriate for predicting dynamic performance (Bazyler et al., 2015; Wilson and Murphy, 1996). However, it has been also proposed that multi-joint isometric tests conducted at specific joint angles may be appropriate to assess dynamic performance (Bazyler et al., 2015). In various athletic populations (i.e., soccer players and wrestlers), strong relationships between isometric strength and performance (i.e., jumping and throwing ability) have been found (Kraska et al., 2009; McGuigan et al., 2006; McGuigan and Winchester, 2008; Stone et al., 2003, 2004). Although previous studies have analyzed the relationship between maximal isometric handgrip strength of the dominant arm and serve velocity (Bonato et al., 2014; Pugh et al., 2003), there is still a paucity of studies analyzing the relationship between maximal isometric strength and different upper and lower limb joints involved in the kinetic chain (i.e., wrist, elbow, shoulder, leg and back). Therefore, the aims of this study were (a) to analyze the relationship between maximum isometric strength levels in different upper and lower limb joints involved in the service kinetic chain and serve velocity in competitive tennis players, and (b) to determine a prediction model based on the relationship between these variables.

\section{Material and Methods}

\section{Participants}

Twelve male high-performance tennis players (mean \pm SD; age: $17.2 \pm 1.0$ years; body height: $180.1 \pm 6.2 \mathrm{~cm}$; body mass: $71.9 \pm 5.6 \mathrm{~kg}$ ) with International Tennis Numbers (ITN) ranging from 1 (elite) to 2 (advanced) volunteered to participate in the study. All the players participated in an average of 20 to 25 hours of training per week, which focused on tennisspecific training (i.e., technical and tactical skills), aerobic and anaerobic training (i.e., on- and offcourt exercises) and strength training. All players had a minimum of five years of prior only tennis- specific training oriented to age category competition (i.e., Under [U]12, U14 and U16). Inclusion criteria for all subjects required each participant to be a healthy tennis player with no history of upper extremity surgery, no shoulder, back or knee pain for the past 12 months and no rehabilitation for the past 12 months. No vigorous physical activity was performed in the 24 hours before testing. All the players were right-handed. No caffeine ingestion was allowed in the 24 hours before testing. Before participating, all subjects provided written informed consent, and experimental procedures as well as potential risks were explained. Parental written informed consent was obtained for subjects under 18 years of age. The scientific committee of the Research and Health Education Foundation of Osona approved the study.

\section{Procedures}

The study was divided into two testing sessions: (a) serve velocity and (b) maximum isometric strength tests, which were performed on the same day, with 30 min rest periods between sessions. On the one hand, this procedure was used to determine to what extent maximum isometric strength related to serve velocity. On the other hand, multiple regression analyses were used to develop models that were most effective at predicting serve velocity. Before any baseline testing, all participants attended two familiarization sessions to introduce the testing procedures and to ensure that any learning effect was minimal for the study measures. To reduce the interference of uncontrolled variables, all the subjects were instructed to maintain their habitual lifestyle and normal dietary intake before and during the study. The subjects were told not to exercise the day before testing and to consume their last (caffeine-free) meal at least three hours before the scheduled test time.

Serve Velocity Testing

Testing was conducted on a hard-surface tennis court (GreenSet surface, Worldwide S.L., Barcelona, Spain) with stable wind conditions (air velocity $<2 \mathrm{~m} \cdot \mathrm{s}^{-1}$ ), using new tennis balls (Head ATP, Spain). Before the serving test, all subjects performed a warm-up protocol (i.e., dynamic movements in the shoulder, plus 8 to 12 slow serves). Each player was instructed to hit two sets of six flat serves (i.e., with a minimum amount of spin) on each side of the court with $60 \mathrm{~s}$ rest 
periods between sets. Only the services that were "in" were registered. In this regard, we assumed that the direction and service target $(\mathrm{T}$, body and wide) significantly affected the execution of the serve (Reid et al., 2011) and this fact might influence the test reliability. Peak ball velocity $\left(\mathrm{km} \cdot \mathrm{h}^{-1}\right)$ was measured in real time by a hand-held radar gun (Stalker Pro, EUA; frequency: $34.7 \mathrm{GHz}$ [Ka-Band] $\pm 50 \mathrm{MHz}$ ). The radar was positioned at the center of the baseline, $4 \mathrm{~m}$ behind the server, aligned with the approximate height of ball contact $(\sim 2.2 \mathrm{~m})$, and pointing down the center of the court. For data analysis, the average values of serves in play were recorded. Subjects were encouraged to hit the ball as hard as possible using a normal tennis serve form. Direct feedback of velocities was provided to encourage maximal effort. Inter-trial reliability for serve velocity was $3.1 \pm 1.1 \%$.

Isometric Strength Testing

Participants were asked to perform nine maximal isometric tests (leg and back extension; wrist and elbow flexion and extension; shoulder flexion and extension, internal and external rotation). Only the dominant arm was tested. Isometric peak force was measured using a strain gauge (MuscleLab 4000e; BoscosystemLab, Rome, Italy). The amplified and calibrated force signal was sampled at $200 \mathrm{~Hz}$. The specific position for each isometric pull was established before each trial with the use of goniometry. Subjects performed three maximal voluntary contractions of 3-5 s duration separated by 1 min (between sets) and $5 \mathrm{~min}$ (between joints) of rest. Strong verbal encouragement was given during every contraction to promote maximal and fast voluntary effort. The isometric mid-thigh pull test was performed in a closed kinetic chain position with a multipower strength machine (Technogym ${ }^{\circledR}$, Italy), the angle selected was $70^{\circ}$ ( $0^{\circ}$ was fully extended). Upper limb positions were tested in a cable jungle machine (Technogym ${ }^{\circledR}$, Italy) with subjects seated. Similar racket grip diameter was selected with the aim of increasing specificity, and special attention was placed on grip comfort during tests to avoid strength feedback inhibition (Shim et al., 2012). In the upper limb tests, participants sat in an upright position with $90^{\circ}$ of flexion at the hip, with the thighs supported and the medial borders of the knees placed together. All participants were restrained by a waist band and two thoracic straps crossing over at the sternum, which together acted to prevent any extraneous movement. Shoulder flexion and extension positions were recorded with the upper extremity flexed to $90^{\circ}$ (Hurd and Kaufman, 2012; Hurd et al., 2011) and the elbow extended, while the shoulder rotation movement was performed with the shoulder abducted and the elbow flexed to $90^{\circ}$. To prevent hip extensor activity from contributing to the isometric peak force of the lumbar extensors, the apparatus seat height was adjusted so that the trunk-thigh angle was $135^{\circ}$ and the shank-thigh angle was $90^{\circ}$. Elbow and wrist tests were performed in a seated position, with the elbow bent at $90^{\circ}$. In both cases, subjects were asked to hold a U-shaped handle linked to the strain gauge in a prone position.

\section{Statistical Analyses}

Mean values $( \pm S D)$ were calculated for all variables. The normality of variable distribution was assessed by the Kolmogorov-Smirnov test. The relationship between quantitative variables was described using Pearson's product-moment correlation coefficients (r). Multiple regression analyses were performed to explain the variance in the serve ball velocity, using the independent variables of upper and lower limb positions. Significance was tested at the $95 \%$ confidence level $(p<\alpha \leq 0.05)$. All statistical analyses were performed using SPSS for Windows 15.0 (SPSS, Inc., Chicago, IL, USA).

\section{Results}

Players hit a total of 144 serves (72 to the left side and 72 to the right side). Mean ( \pm SD) serve velocity and serves considered good are shown in Table 1.

The relationships between maximal isometric strength values and serve velocity for each of the measurements are shown in Table 2. Serve velocity demonstrated a strong positive relationship with shoulder internal rotation $(r=$ $0.67 ; p<0.05)$. None of the other quantitative variables analyzed was significantly correlated with serve velocity in any of the analyzed positions, with Pearson's $\mathrm{r}$ coefficients ranging from low to moderate $(r=0.36-0.53)$.

Multiple regression analyses were conducted to evaluate how maximal isometric strength values predicted serve velocity. Leg and 
back extension, wrist and elbow flexion and extension, shoulder flexion and extension, and internal and external rotation were used as independent or predictor variables, and peak ball serve velocity as a dependent or predicted variable. The linear combination of shoulder flexion and internal rotation was significantly related to serve velocity $(\mathrm{F}(2,8)=4.784, p<0.05)$. The multiple correlation coefficient was 0.74 , indicating that approximately $55 \%$ of the variance of the serve velocity could be accounted for by the linear combination of shoulder flexion and internal rotation maximal isometric strength. The regression equation for predicting the serve velocity was: Serve velocity $=143.86+(0.07 \mathrm{x}$ shoulder internal rotation $)+(0.068 \times$ shoulder flexion).

When including wrist flexion, the multiple correlation coefficient was 0.76 , indicating that the proportion of variance in serve velocity explained by the model increased only to $58 \%$. When the other strength variables were added to the model, their ability to predict serve velocity was reduced.

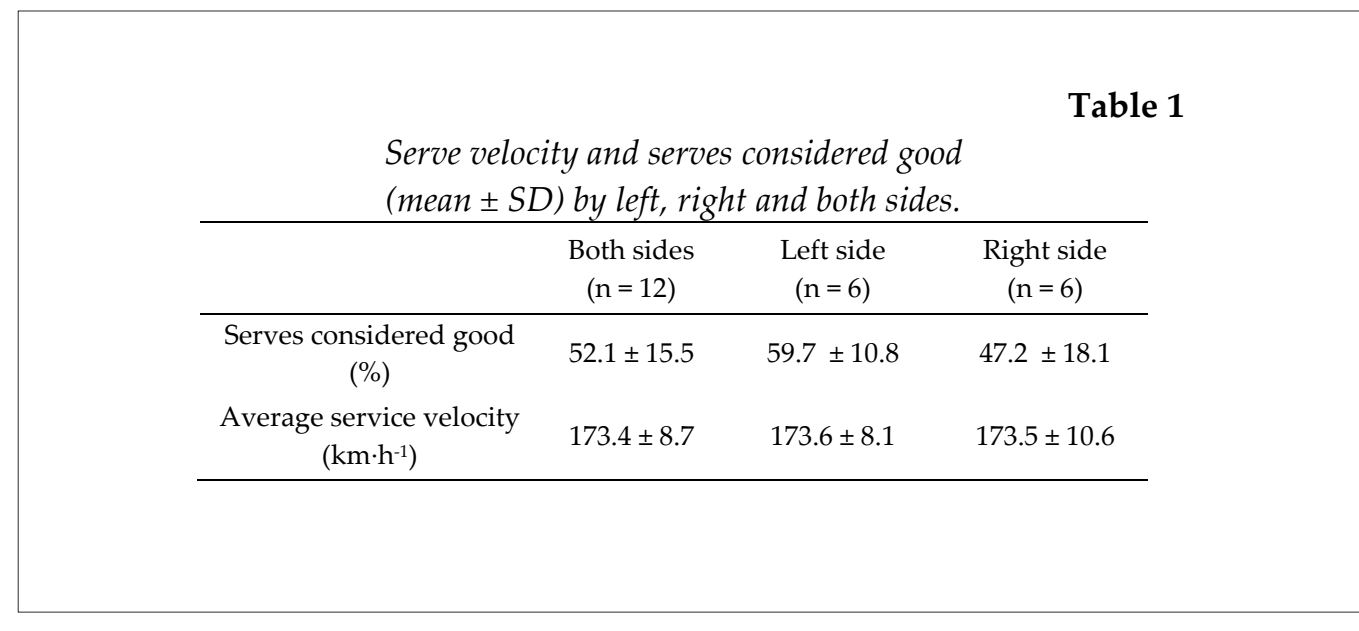

\begin{tabular}{|c|c|c|c|}
\hline \multicolumn{4}{|c|}{$\begin{array}{l}\text { Maximum isometric strength variables (mean } \pm S D \text { ) } \\
\text { and correlation coefficient }(r) \text { between serve velocity. }\end{array}$} \\
\hline \multirow[t]{2}{*}{ Variables } & \multirow{2}{*}{$\begin{array}{l}\text { Maximum isometric } \\
\text { strength } \\
(\mathrm{N})\end{array}$} & \multicolumn{2}{|c|}{$\begin{array}{l}\text { Average serve velocity } \\
\left(\mathrm{km} \cdot \mathrm{h}^{-1}\right)\end{array}$} \\
\hline & & $r$ & $p$ \\
\hline \multicolumn{4}{|l|}{ Extension } \\
\hline Leg and back & $1351 \pm 469.6$ & 0.47 & 0.121 \\
\hline Wrist & $157.4 \pm 47.9$ & 0.37 & 0.265 \\
\hline Elbow & $199.2 \pm 66.6$ & 0.53 & 0.144 \\
\hline Shoulder & $126.1 \pm 29.4$ & 0.48 & 0.119 \\
\hline \multicolumn{4}{|l|}{ Flexion } \\
\hline Wrist & $260.7 \pm 65.3$ & 0.52 & 0.085 \\
\hline Elbow & $219.4 \pm 64.3$ & 0.36 & 0.377 \\
\hline Shoulder & $197.0 \pm 39.1$ & 0.57 & 0.054 \\
\hline \multicolumn{4}{|l|}{ Rotation } \\
\hline $\begin{array}{l}\text { Shoulder external } \\
\text { rotation }\end{array}$ & $107.3 \pm 30.1$ & 0.50 & 0.117 \\
\hline Shoulder internal rotation & $197.8 \pm 62.2$ & 0.67 & 0.023 \\
\hline
\end{tabular}




\section{Discussion}

The main finding of this study was that maximum isometric strength of shoulder internal rotation was strongly related to serve velocity and that shoulder flexion and internal rotation maximum isometric strength seemed to be good predictors of ball speed on a serve, explaining $55 \%$ of the variance in serve velocity.

The importance of maximum isometric strength in different sports has been previously demonstrated (McGuigan and Winchester, 2008). To the best of our knowledge, this is the first study that has related maximum isometric strength of different upper and lower body joints to serve velocity in competitive tennis players. A strong significant relationship was found between maximum isometric strength levels in shoulder internal rotation and serve velocity $(\mathrm{r}=0.67 ; \mathrm{p}<$ 0.05), highlighting an important role that the shoulder internal rotation plays in the serve motion. In this regard, shoulder internal rotation is considered a key element to developing high racket velocities and hence, fast serves (Elliott, 2006; Elliott et al., 1995; Elliott et al., 2003). In contrast to these results, when strength testing was conducted using isokinetic dynamometry, the relationship between shoulder internal rotation and ball serve velocity was found to be low and not statistically significant (Cohen et al., 1994; Pugh et al., 2003; Signorile et al., 2005). This emphasizes the differences between static strength testing (the joint angle and muscle length do not change during contraction) and dynamic strength testing with limb movements at constant velocity around the joint (the velocity of movement is maintained constant by a special dynamometer) (Baltzopoulos and Brodie, 1989). On the contrary, if we compare the contribution of speed of shoulder ( $40 \%)$ and wrist $(\sim 30 \%)$ joints to the linear racket speed prior to impact (Elliot et al., 1995; Gordon and Dapena, 2006; Martin et al., 2013; Sprigings et al., 1994), this contribution coincides with the coefficients of determination $\left(r^{2}\right)$ between maximum isometric strength of the internal rotation $(45 \%)$, wrist flexion $(27 \%)$ and serve velocity, showing the similarities between these two different methods (maximum isometric strength vs speed of joints). None of the other quantitative variables analyzed (maximum strength level in leg and back, wrist, elbow and shoulder extension; wrist, elbow and shoulder flexion; and shoulder external rotation) was significantly correlated with serve velocity ( $\mathrm{r}=$ $0.36-0.57)$. Although these joints and movements are included as a part of the service kinetic chain (Elliott, 2006), the isolated variables, without being related with other predictor variables, are not good predictors of ball speed during the tennis serve. This fact denotes the limitations of evaluating isolated joints and movements related with serve speed; moreover, it emphasizes the importance of an efficient kinetic chain for a high speed tennis serve. The kinetic chain of the tennis service starts with the feet and knees generating ground reaction forces that can be transferred up to legs, the trunk/back and the shoulder to the elbow joint and finally to the wrist and the hand (Bonato et al., 2015; Eygendaal et al., 2007). This complex and coordinated action implies a synchronized movement summating forces by one joint (e.g., shoulder) to the next one (e.g., elbow and wrist) throughout all the joints of the kinetic chain and out into the ball. This implies inter-muscular coordination (magnitude and timing) of agonist, synergist and antagonist muscles during the powerful movement (Cormie et al., 2014).

The multivariate analyses used to predict player's serve velocity have showed that approximately half $(55 \%)$ of the variability in serve velocity can be explained by shoulder internal rotation and shoulder flexion, suggesting that isometric testing provides an acceptable indication of serve velocity in tennis. In this regard, Signorile et al. (2005) found that the multiple regression model using diagonal throwing peak torque was predictive of peak serve speed $\left(\mathrm{r}^{2}=0.69 ; \mathrm{p}<0.0001\right)$, and this complex movement (a diagonal throw) includes shoulder flexion and internal rotation. The multivariate analyses conducted previously using isokinetic dynamometry did not include shoulder internal rotation in the best prediction model (Cohen et al., 1994; Pugh et al., 2003), again highlighting the differences between both methods of strength testing (isometric versus isokinetic dynamometry). This previous study found that wrist flexion and elbow extension torque production were highly related to serve velocity ( $p<0.01$ ) (Cohen et al., 1994) and that only around $19 \%$ of the variance in ball speed was accounted for by knee extension, shoulder 
rotation and grip strength (Pugh et al., 2003).

The wrist flexion slightly assists in generating high velocity, increasing the variance explained in serve velocity by only $3 \%$, indicating that it is not one of the main contributing joint. This low contribution may be due to the fact that the muscle chain in the upper limb will follow proximal to distal order of activation (Elliott et al., 2003), and the wrist represents the final link of this kinetic chain, not creating the power, but transferring the final ball speed. The other variables analyzed (leg and back, wrist and elbow extension; elbow flexion; and shoulder external rotation) reduced the predictive strength of our equation, indicating that these variables were not directly involved in the acceleration phase of the racket to the ball. The rest of the variance in serve velocity may be explained by the multifactorial nature of the tennis serve motion as well as the fact that strength is not the only factor involved in producing ball speed during the tennis serve. Ball speed depends on a combination of several factors such as technique, coordination and flexibility (Cohen et al., 1994; Pugh et al., 2003; Reid and Schneiker, 2008). Furthermore, there are other muscular groups that can contribute to serve velocity. For example, it may be possible to increase the predictive ability of the model by introducing an assessment of trunk strength. Experienced tennis players effectively use the kinetic chain (i.e., rotate, extend and flex the trunk to produce force) via a lower extremity muscle activation to provide a stable base (Kovacs and Ellenbecker, 2011a; Kovacs and Ellenbecker, 2011b). Additionally, the magnitude of the angular momentum generated by the trunk in the frontal plane during the serve helps distinguish high-speed from low-speed servers (Bahamonde, 2000).

Maximum isometric shoulder internal rotation and flexion seem to be good predictors of serve velocity. Therefore, tennis coaches and physical trainers have a choice of which static muscular actions to use in their strength training aimed to improve serve velocity. In this regard, isometric strength training has been associated with significant improvements in dynamic strength, although it only produces adaptations at the specific trained joint angle, with less transfer to other muscle lengths (Folland et al., 2005). The tennis serve is a dynamic movement where the summation of forces from the ground up through the kinetic chain is sustained by a stretchshortening cycle, and the total body perspective is just as important as individual segments in isolation (Kovacs and Ellenbecker, 2011b). Therefore, on the one hand, maximal isometric strength training should only be an additional method and should be combined with dynamic and ballistic methods (e.g., elastic tubing, medicine ball exercises, resistance training or lightweight dumbbell training). On the other hand, it is not recommended that coaches perform only analytical strength exercises (i.e., shoulder rotation or shoulder flexion). In order to achieve better results, coaches should consider using training methods that allow for involving these two movements within the specific kinetic chain of service. To develop stroke velocity, other authors have recommended the use of medicine ball throws (plyometrics) (Earp and Kraemer, 2010; Genevois et al., 2013; Reid and Schneiker, 2008; Wakeham and Jacobs, 2009) or cable pulley machines (Keiser pneumatic devices) (Kovacs and Ellenbecker, 2011b; Roetert et al., 2009). These exercises make it possible to incorporate shoulder internal rotation and shoulder flexion into the kinetic chain (i.e., an overhead diagonal cable or a medicine ball throw). Exercises of this kind may complement generic strength training and allow to meet sport-specific demands (i.e., the plane of movement, velocity and body positioning during the strokes) (Earp and Kraemer, 2010).

There are certain limitations of this study. First, as previously discussed, to improve the predictive capacity of the model, it would be necessary to introduce other variables such as technique, coordination, flexibility or other strength variables such as trunk rotation or core stability. Second, isometric testing was conducted using static positions and this method cannot replicate all joint angles, specific tennis movements and the rotation velocities of segments in stroke production (Murphy and Wilson, 1997). Moreover, interference of uncontrolled variables such as different service techniques used (i.e., foot-up and foot-back), the type of a racket and the type and tension of strings on the rackets used by players, could have a direct impact on serve velocity (Bower and Cross, 2005; Lees, 2003). Finally, the effectiveness of tennis serve is not only determined by the ball 
speed, there are important additional performance indicators such as the ball rotation or spin (topspin or slice serves) and accuracy (Abrams et al., 2011; Elliot et al., 1995).

In conclusion, the present study showed that the maximum isometric strength level in the shoulder internal rotation was strongly related to serve velocity in high-performance tennis players and a large part of the variability in serve velocity could be explained by maximum isometric strength levels in shoulder internal rotation and shoulder flexion.

\section{Acknowledgements}

This work was supported by Institut Nacional d'Educació Física de Catalunya. The authors thank all the players and coaches for their enthusiastic participation. They would also like to thank the Centre Internacional de Tennis of the Catalan Tennis Federation. The authors gratefully acknowledge the technical assistance of Andreu Fuster, Margalida Mas and Abraham Batalla during the experiments.

\section{References}

Abrams GD, Sheets AL, Andriacchi TP, Safran MR. Review of tennis serve motion analysis and the biomechanics of three serve types with implications for injury. Sports Biomech, 2011; 10: 378-390

Bahamonde RE. Changes in angular momentum during the tennis serve. J Sports Sci, 2000; 18: 579-92

Baltzopoulos V, Brodie DA. Isokinetic dynamometry. Applications and limitations. Sports Med, 1989; 8: 101116

Bazyler CD, Beckham GK, Sato K. The use of the isometric squat as a measure of strength and explosiveness. J Strength Cond Res, 2015; 29: 1386-1392

Bonato M, Maggioni MA, Rossi C, Rampichini S, La Torre A, Merati G. Relationship between anthropometric or functional characteristics and maximal serve velocity in professional tennis players. J Sports Med Phys Fitness, 2015; 55: 1157- 1165

Bower R, Cross R. String tension effects on tennis ball rebound speed and accuracy during playing conditions. J Sports Sci, 2005; 23: 765-771

Cardoso Marques MA. Strength training in adult elite tennis players. Strength Cond J, 2005; 27: 34-41

Cohen DB, Mont MA, Campbell KR, Vogelstein BN, Loewy JW. Upper extremity physical factors affecting tennis serve velocity. Am J Sports Med, 1994; 22: 746-750

Cormie P, McGuigan MR, Newton RU. Developing maximal neuromuscular power. Part 1 - Biological basis of maximal power production. Sports Med, 2011; 41: 17-38

Earp JE, Kraemer WJ. Medicine ball training implications for rotational power sports. Strength Cond J, 2010; 32: $20-25$

Elliott B. Biomechanics and tennis. Br J Sports Med, 2006; 40: 392-396

Elliott B, Marshall RN, Noffal GJ. Contributions of upper limb segment rotations during the power serve in tennis. J Appl Biomech, 1995; 11: 433-442

Elliott B, Reid M, Crespo M. ITF biomechanics of advanced tennis. London: ITF Ltd; 2003

Eygendaal D, Rahussen FTG, Diercks RL. Biomechanics of the elbow joint in tennis players and relation to pathology. Br J Sports Med, 2007; 41: 820-823

Fernandez-Fernandez J, Ellenbecker T, Sanz-Rivas D, Ulbricht A, Ferrauti A. Effects of a 6-week junior tennis conditioning program on service velocity. J Sports Sci Med, 2013; 12: 232-239

Folland JP, Hawker K, Leach B, Little T, Jones D. Strength training: Isometric training at a range of joint angles versus dynamic training. J Sports Sci, 2005; 23: 817-824

Genevois C, Frican B, Creveaux T, Hautier C, Rogowski I. Effects of two training protocols on the forehand 
drive performance in tennis. J Strength Cond Res, 2013; 27: 677-682

Gillet E, Leroy D, Thouvarecq R, Stein JF. A notational analysis of elite tennis serve and serve-return strategies on slow surface. J Strength Cond Res, 2009; 23: 532-539

Gordon BJ, Dapena J. Contributions of joint rotations to racquet speed in the tennis serve. J Sports Sci, 2006; 24: 31-49

Hurd WJ, Kaplan KM, El Attrache NS, Jobe FW, Morrey BF, Kaufman KR. A profile of glenohumeral internal and external rotation motion in the uninjured high school baseball pitcher. J Athl Train, 2011; 46: 289295

Hurd WJ, Kaufman KR. Glenohumeral rotational motion and strength and baseball pitching biomechanics. J Athl Train, 2012; 47: 247-256

Jayanthi N, Esser S. Racket sports. Curr Sports Med Rep, 2013; 12: 329-336

Kovacs M, Ellenbecker T. An 8-stage model for evaluating the tennis serve: Implications for performance enhancement and injury prevention. Sports Health, 2011a; 3: 504-513

Kovacs M, Ellenbecker T. A performance evaluation of the tennis serve: Implications for strength, speed, power, and flexibility training. Strength Cond J, 2011b; 33: 22-30

Kraemer WJ, Hakkinen K, Triplett-Mcbride NT, Fry AC, Koziris LP, Ratamess NA, Bauer JE, Volek JS, McConnell T, Newton RU, Gordon SE, Cummings D, Hauth J, Pullo F, Lynch JM, Fleck SJ, Mazzetti SA, Knuttgen HG. Physiological changes with periodized resistance training in women tennis players. Med Sci Sports Exerc, 2003; 35: 157-168

Kraska JM, Ramsey MW, Haff GG, Fethke N, Sands WA, Stone ME, Stone MH. Relationship between strength characteristics and unweighted and weighted vertical jump height. Int J Sports Physiol Perform, 2009; 4: 461-73

Lees A. Science and the major racket sports: A review. J Sports Sci, 2003; 21: 707-732

Martin C, Kulpa R, Delamarche P, Bideau B. Professional tennis players' serve: correlation between segmental angular momentums and ball velocity. Sports Biomech, 2013; 12: 2-14

McGuigan MR, Winchester JB. The relationship between isometric and dynamic strength in college football players. J Sports Sci Med, 2008; 7: 101-105

McGuigan MR, Winchester JB, Erickson T. The importance of isometric maximum strength in college wrestlers. J Sports Sci Med, 2006; 5: 108-113

Murphy AJ, Wilson GJ. The ability of tests of muscular function to reflect training-induced changes in performance. J Sports Sci, 1997; 15: 191-200

Pugh SF, Kovaleski JE, Heitman RJ, Gilley WF. Upper and lower body strength in relation to ball speed during a serve by male collegiate tennis players. Percept Mot Skills, 2003; 97: 867-872

Reid M, Schneiker K. Strength and conditioning in tennis: Current research and practice. J Sci Med Sport, 2008; 11: 248-256

Reid M, Whiteside D, Elliot B. Serving to different locations: set-up, toss, and racket kinematics of the professional tennis serve. Sports Biomech, 2011; 10: 407-414

Roetert EP, Kovacs M, Knudson D, Groppel JL. Biomechanics of the tennis groundstrokes: Implications for strength training. Strength Cond J, 2009; 31: 41-49

Shim JK, Karol S, Kim YS, Seo NJ, Kim YH, Kim Y, Yoon BC. Tactile feedback plays a critical role in maximum finger force production. J Biomech, 2012; 45: 415-20

Signorile JF, Sandler DJ, Smith WN, Stoutenberg M, Perry AC. Correlation analyses and regression modeling between isokinetic testing and on-court performance in competitive adolescent tennis players. $J$ Strength Cond Res, 2005; 19: 519-526

Sprigings E, Marshall R, Elliott B, Jennings L. A three dimensional kinematic method for determining the 
effectiveness of arm segment rotations in producing racket-head speed. J Biomech, 1994; 27: 245-254

Stone MH, Sanborn K, O'Bryant HS, Hartman M, Stone ME, Proulx C, Ward B, Hruby J. Maximum strengthpower-performance relationships in collegiate throwers. J Strength Cond Res, 2003; 17: 739-45

Stone MH, Sands WA, Carlock J, Callan S, Dickie D, Daigle K, Cotton J, Smith SL, Hartman M. The importance of isometric maximum strength and peak rate-of-force development in sprint cycling. $J$ Strength Cond Res, 2004; 18: 878-84

Treiber FA, Lott J, Duncan J, Slavens G, Davis H. Effects of Theraband and lightweight dumbbell training on shoulder rotation torque and serve performance in college tennis players. Am J Sports Med, 1998; 26: $510-5$

Wakeham T, Jacobs R. Preseason strength and conditioning for collegiate tennis players. Strength Cond J, 2009; 31: 86-93

Wilson GJ, Murphy AJ. The use of isometric test of muscular function in athletic assessment. Sports Med, 1996; 22: 19-37

\section{Corresponding author:}

\section{Ernest Baiget}

Postal address: University of Vic - Central University of Catalonia, Sagrada Família 7, 08500, Vic, Spain Telephone: +34 938816164

Fax: +34 938891063

E-mail address: ernest.baiget@uvic.cat 